\title{
تاثير المبيد Oxamyl في الوراثة الخلوية للخلايا اللمفاوية للام المحيطي في الانسان
}

\section{Effect of Oxamyl on Cytogenetics of human peripheral lymphocytes}

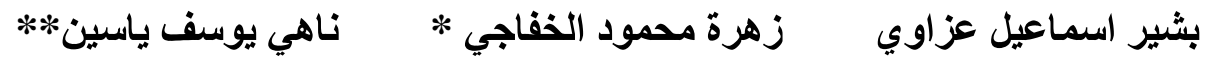 \\ مركز بحوث التقنيات الاحيائية / جامعة النهرين

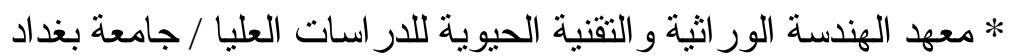

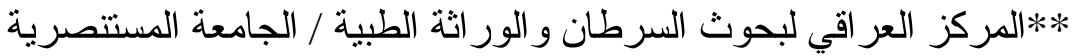

\section{Basheer I . Azawei Zahra M. Al-Khafaji* Nahi Y. Yassein **}

Biotechnology Research Center/ Al- Nahrain University

*Institue of Genetic Engineering and Biotechnology for Postgraduate

Studies / University of Baghdad

**The Iraqi Center for Cancer Research and Medical Genetics /

University of Mustansseryia

درس تأثير احل مبيدات الكاربامات وهو Oxamyl على بعض مؤشرات الوراثذة الخلويـة في لمفاويـات الدام

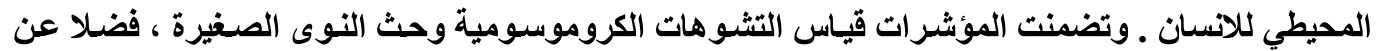

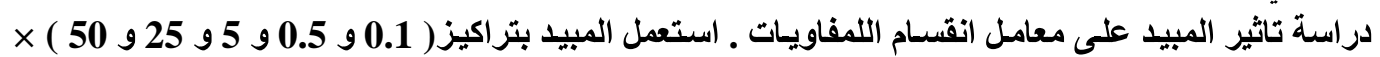

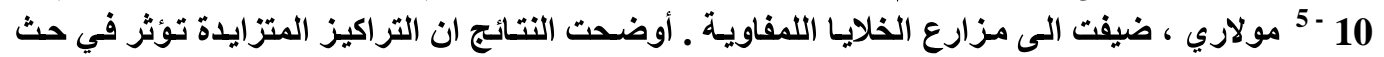

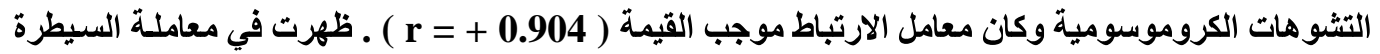

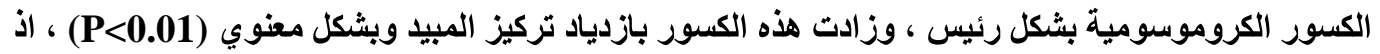

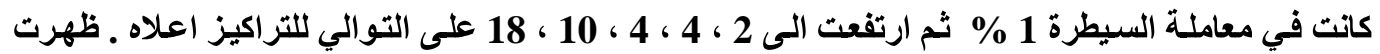

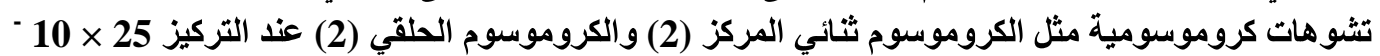

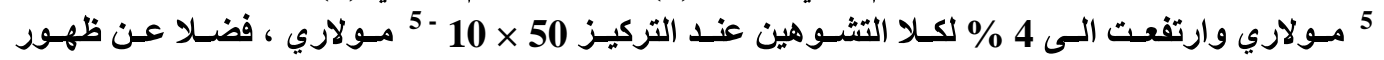

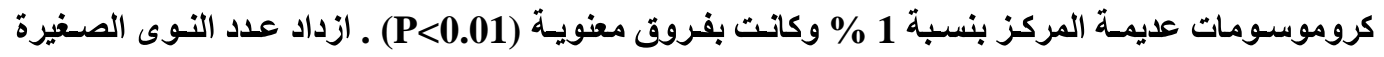

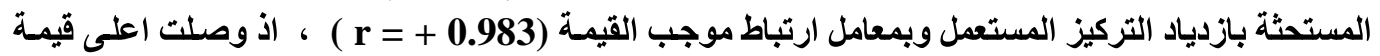

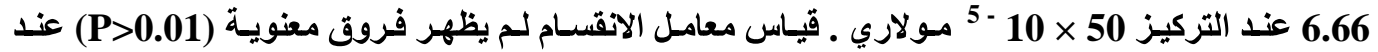

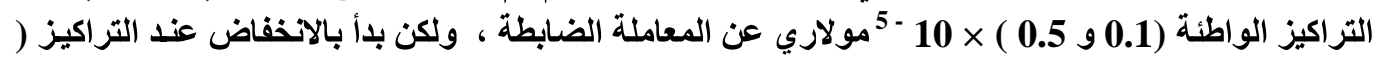

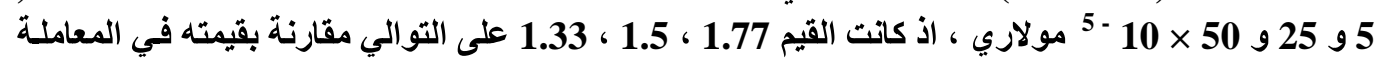
الضابطة 2.5 وباختلاف معنوي 50 (P>0.01 )

\section{Abstract}

The effect of of carbamate pesticides (Oxamyl) on some parameters of cytogenetics of human peripheral blood lymphocytes was studied. These parameters were induction of chromosomal aberrations (CAs) and it's types; and formation of Micronuclei (Mn); in addition to estimating the mitotic index (MI) of cultured cells. The concentrations used were $(0.1,0.5,5,25,50) \times 10^{-5} M^{\text {which }}$ 
were added to lymphocyte cultures. Results indicated that the concentrations induced the CAs as the concentrations increased with positive correlation coefficient $(r=+0.904)$. Abnormalities in the control treatment were mainly as chromosomal breaks, these increased to $2,4,4,10,18$ respectively and were with significant differences compared to control treatment $(\mathbf{P}<0.01)$. Dicentric chromosomes appeared with $2 \%$ level at concentration $25 \times 10^{-5} \mathrm{M}$, the latter abnormality raised to $4 \%$ at concentration $50 \times 10^{-5} \mathrm{M}$ in addition to the appearance of acentric chromosomes $(1 \%)$, all of them were with significant differences $(\mathbf{P}<\mathbf{0 . 0 1})$. Micronuclei $(\mathrm{Mn})$ increased in cells as the concentrations increased with positive correlation coefficient $(r=+0.983)$, and the highest value recorded at concentration $50 \times 10^{-5 \mathrm{M}}(6.66 \%)$. MI had no significant difference from the control treatment $(\mathbf{P}>\mathbf{0 . 0 1})$, but started to decline at concentrations 5 , $25,50 \times 10^{-5} \mathrm{M}$ and the values were $1.77,1.5,1.33$ respectively compared to control treatment $(2.5)$ with significant difference $(\mathbf{P}<0.01)$.

المقدمة

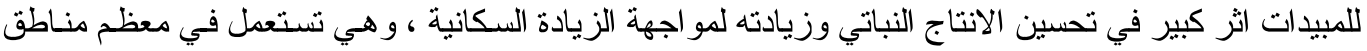

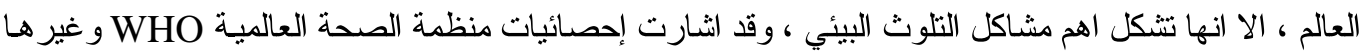

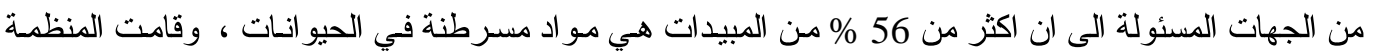

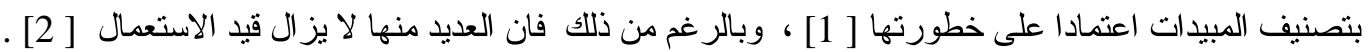

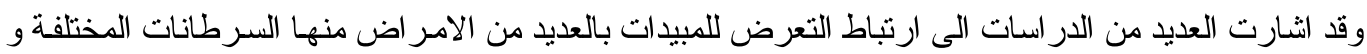

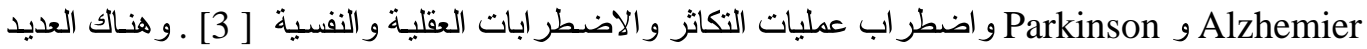

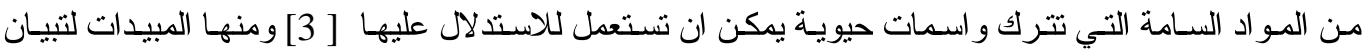
خطر ها. فضلا عن ذلك يلاحظ ان عدد المبيدات في ازدياد و التي تحاول استهداف مو اقع معينة في الآفات الزر اعية للتغلب

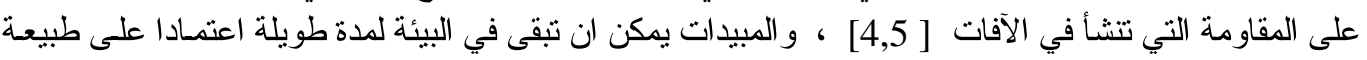
المبيد التركيبية و الظروف البيئية [ [6,7] ـ

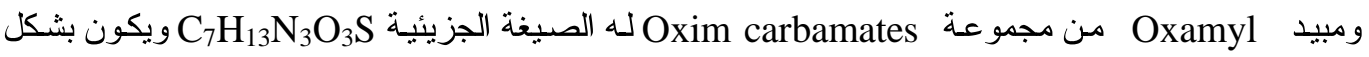

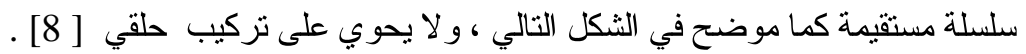

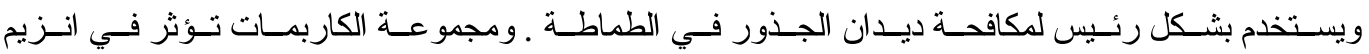

\section{$\left(\mathrm{CH}_{3}\right)_{2} \mathrm{NCOC}=\mathrm{NOCONHCH}_{3}$ $\mathrm{SCH}_{3}$}

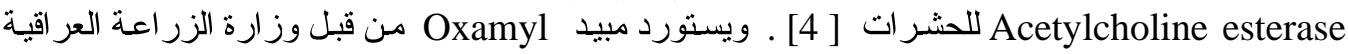
[ 8] ولكن دون اجر اء الفحوص اللازمة لسلامة استعماله.

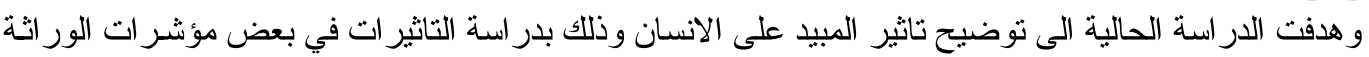

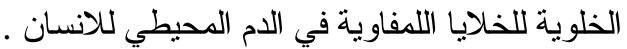

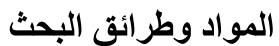
أجريت الدر اسة في المركز العر اقي لبحوث السرطان و الور اثة الطبية / الجامعة المستتصرية / بغداد ـ العر اق ـ

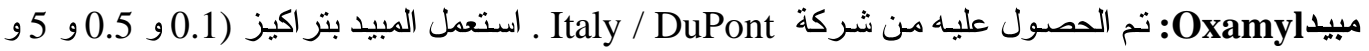

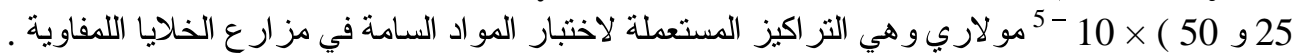

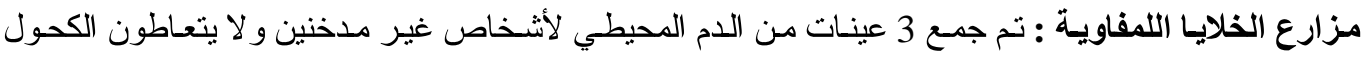

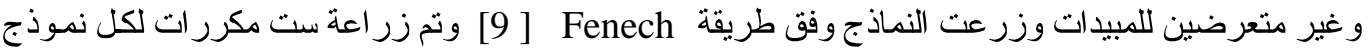

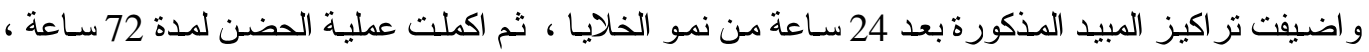

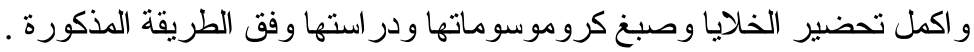


تقتية التحزيم G - Banding technique : استعملت في صبخ الكروموسومات وفق طريقة] 10] لتحديد

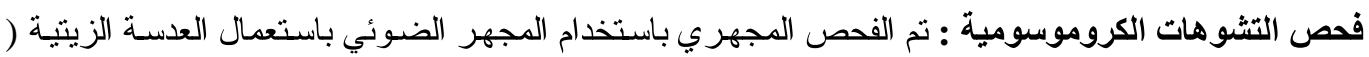

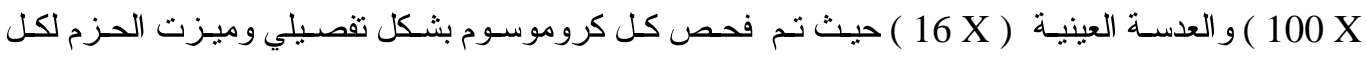
كروموسوم وحسبت عدد التشوهات في ( 100 ) خلية في الطور الاستو ائي ( Metaphase ) من انقسام الخلية

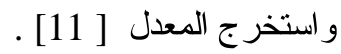

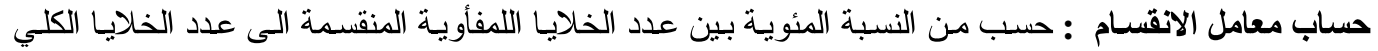

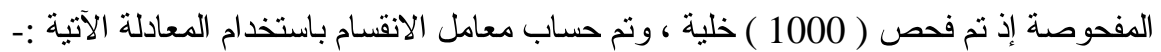

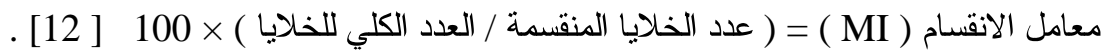

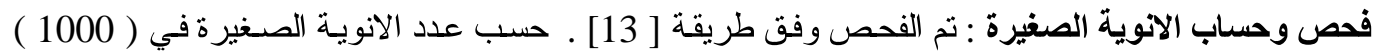

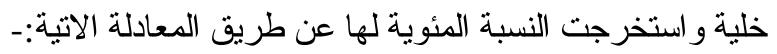

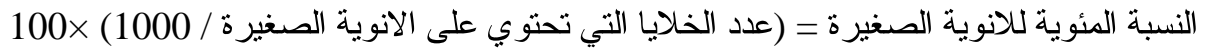

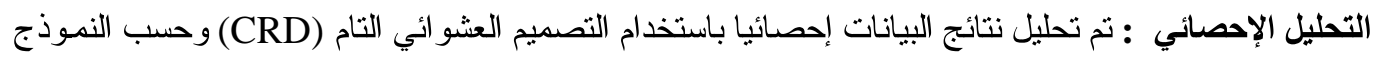

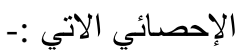

$$
Y i j=M+T i+e i j
$$

$$
\begin{aligned}
& \text { حيث تمثل Yij : الصفة المدروسة } \\
& \text { M : المتوسط العام : M } \\
& \text { ( C = 1-5 : تاثير المعاملة : Ti }
\end{aligned}
$$

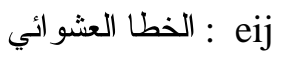

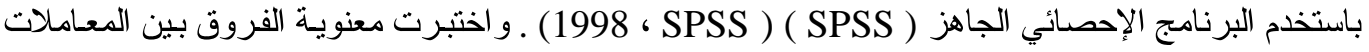

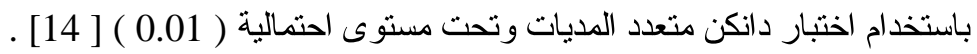

النتائج والمناقشة الثنة تعد در اسة الو اسمات الحيوية Biomarkers التي تتركها المواد السامة ور اثيا من الوسائل الجيدة لتقدير السمية

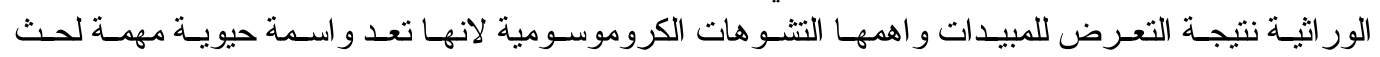

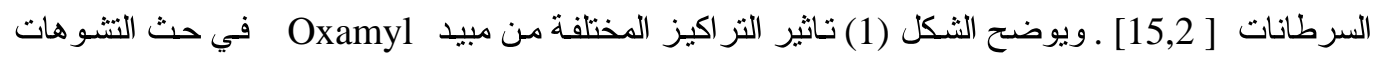

$$
\text { الكروموسومية }
$$

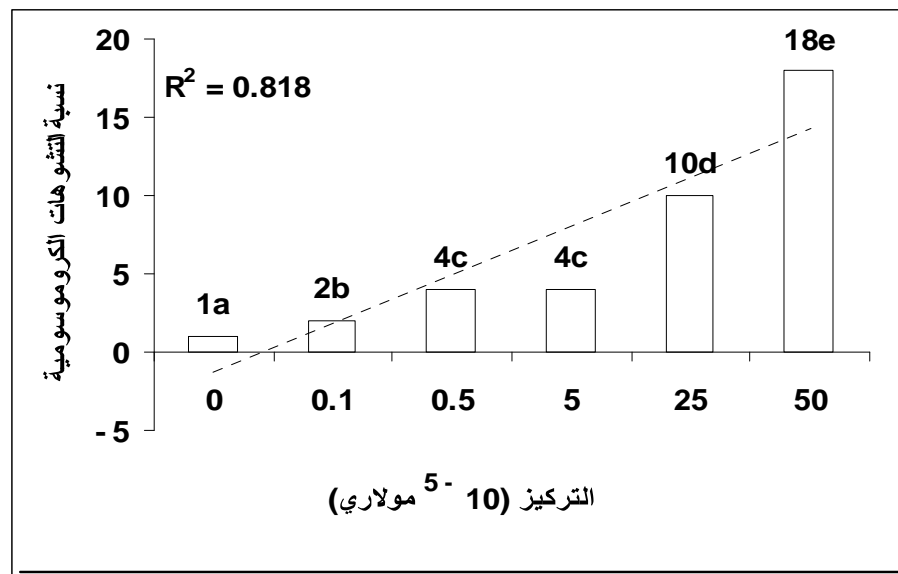

الثكل ( 1) : تاثير مبيدمa في استحثاث التشوهات الكروموسومية في الخلايا اللمفاوية

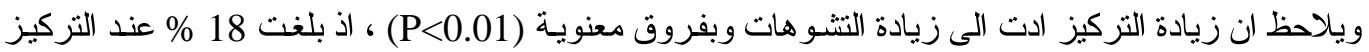

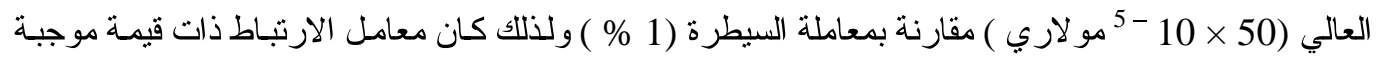

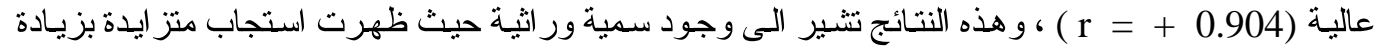

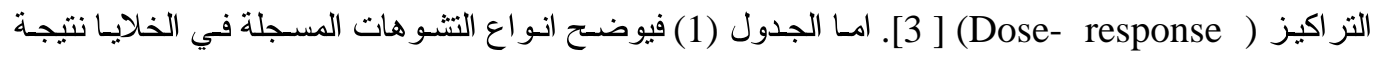




\section{الجدول (1): تاثير التراكيز المختلفة من مبيد اوكساميل (Oxamyl) في استحثاث التشوهات الكروموسومية في خلايا الام اللمفاوية}

\begin{tabular}{|c|c|c|c|c|c|c|c|c|}
\hline \multicolumn{9}{|c|}{ التشوهات الكروموسومية } \\
\hline الكسور & المركزي & المركز & الحذف & الحلقي & الانقلاب & الانتقال & التضاعف & $\begin{array}{c}\text { التزكيز } \\
M \times 10^{-5}\end{array}$ \\
\hline $\begin{array}{c}\mathrm{a} \\
1 \pm 0.06\end{array}$ & $\begin{array}{c}\mathrm{a} \\
0 \pm 0\end{array}$ & $\begin{array}{c}\mathrm{a} \\
0 \pm 0\end{array}$ & $\begin{array}{c}\mathrm{a} \\
0 \pm 0\end{array}$ & $\begin{array}{c}\mathrm{a} \\
0 \pm 0\end{array}$ & $\begin{array}{c}\mathrm{a} \\
0 \pm 0\end{array}$ & $\begin{array}{c}\mathrm{a} \\
0 \pm 0\end{array}$ & $\begin{array}{c}\mathrm{a} \\
0 \pm 0\end{array}$ & السيطرة \\
\hline $\begin{array}{c}\mathrm{b} \\
2 \pm 0.12\end{array}$ & $\begin{array}{c}\mathrm{a} \\
0 \pm 0\end{array}$ & $\begin{array}{c}\mathrm{a} \\
0 \pm 0\end{array}$ & $\begin{array}{c}\mathrm{a} \\
0 \pm 0\end{array}$ & $\begin{array}{c}\mathrm{a} \\
0 \pm 0\end{array}$ & $\begin{array}{c}\mathrm{a} \\
0 \pm 0\end{array}$ & $\begin{array}{c}\mathrm{a} \\
0 \pm 0\end{array}$ & $\begin{array}{c}\mathrm{a} \\
0 \pm 0\end{array}$ & 0.1 \\
\hline $\begin{array}{c}c \\
4 \pm 0.14\end{array}$ & $\begin{array}{c}\mathrm{a} \\
0 \pm 0\end{array}$ & $\begin{array}{c}\mathrm{a} \\
0 \pm 0\end{array}$ & $\begin{array}{c}\mathrm{a} \\
0 \pm 0\end{array}$ & $\begin{array}{c}\mathrm{a} \\
0 \pm 0\end{array}$ & $\begin{array}{c}\mathrm{a} \\
0 \pm 0\end{array}$ & $\begin{array}{c}\mathrm{a} \\
0 \pm 0\end{array}$ & $\begin{array}{c}\mathrm{a} \\
0 \pm 0\end{array}$ & 0.5 \\
\hline $\begin{array}{c}c \\
4 \pm 0.14\end{array}$ & $\begin{array}{c}\mathrm{a} \\
0 \pm 0\end{array}$ & $\begin{array}{c}\mathrm{a} \\
0 \pm 0\end{array}$ & $\begin{array}{c}\mathrm{a} \\
0 \pm 0\end{array}$ & $\begin{array}{c}\mathrm{a} \\
0 \pm 0\end{array}$ & $\begin{array}{c}\mathrm{a} \\
0 \pm 0\end{array}$ & $\begin{array}{c}\mathrm{a} \\
0 \pm 0\end{array}$ & $\begin{array}{c}\mathrm{a} \\
0 \pm 0\end{array}$ & 5 \\
\hline $\begin{array}{c}\mathrm{d} \\
6 \pm 0.24\end{array}$ & $\begin{array}{c}\mathrm{b} \\
2 \pm 0.12\end{array}$ & $\begin{array}{c}\mathrm{a} \\
0 \pm 0\end{array}$ & $\begin{array}{c}\mathrm{a} \\
0 \pm 0\end{array}$ & $\begin{array}{c}\mathrm{b} \\
2 \pm 0.12\end{array}$ & $\begin{array}{c}\mathrm{a} \\
0 \pm 0\end{array}$ & $\begin{array}{c}\mathrm{a} \\
0 \pm 0\end{array}$ & $\begin{array}{c}\mathrm{a} \\
0 \pm 0\end{array}$ & 25 \\
\hline $\begin{array}{c}\mathrm{e} \\
9 \pm 0.2\end{array}$ & $\begin{array}{c}c \\
c \\
4 \pm 0.14\end{array}$ & $\begin{array}{c}\mathrm{b} \\
1 \pm 0.06\end{array}$ & $\begin{array}{c}\mathrm{a} \\
0 \pm 0\end{array}$ & $\begin{array}{c}c c \\
4 \pm 0.14\end{array}$ & $\begin{array}{c}\mathrm{a} \\
0 \pm 0\end{array}$ & $\begin{array}{c}\mathrm{a} \\
0 \pm 0\end{array}$ & $\begin{array}{c}\mathrm{a} \\
0 \pm 0\end{array}$ & 50 \\
\hline
\end{tabular}

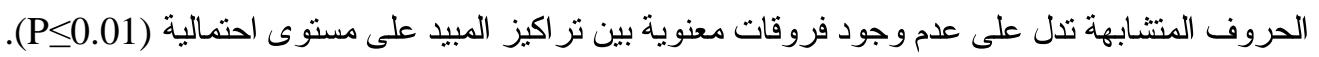

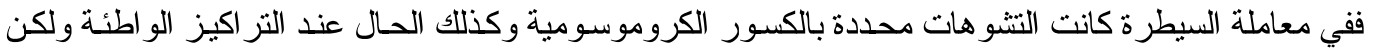

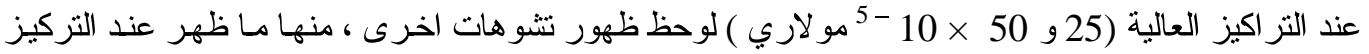

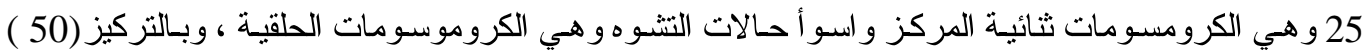

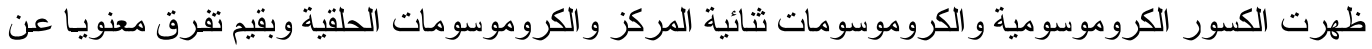

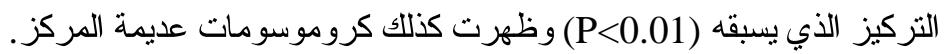

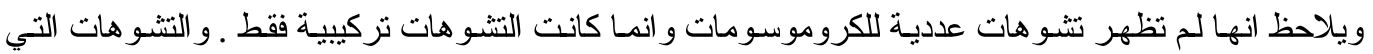

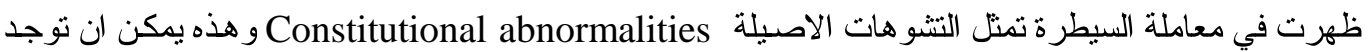

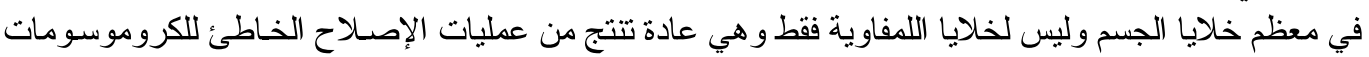

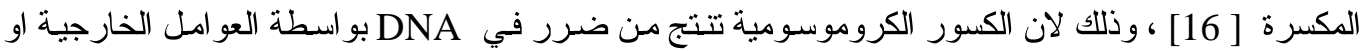

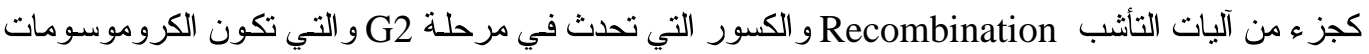

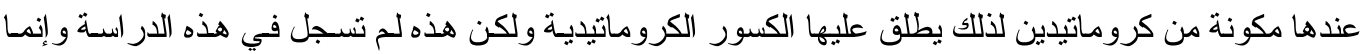

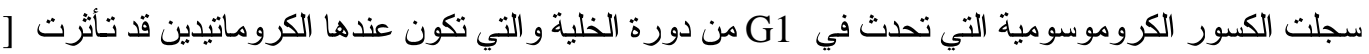

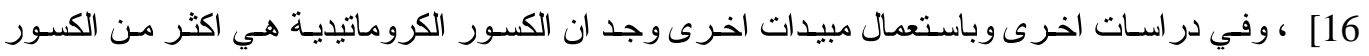

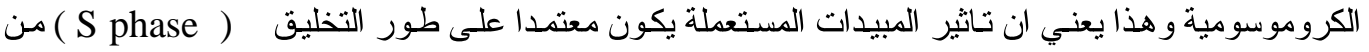

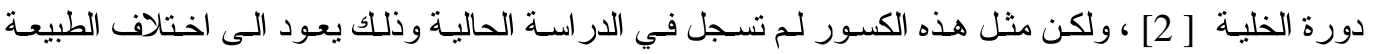

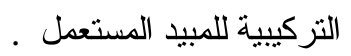

وتعد در اسة الحزم الكروموسومية وسيلة فاعلة في تحديد تاثير المبيدات على المستوى الجزيئي [ 17 ] ، وذلك

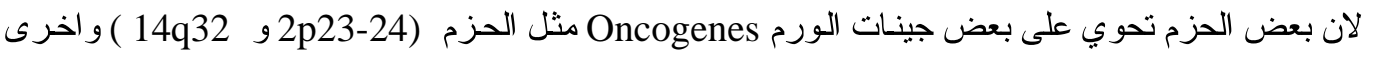

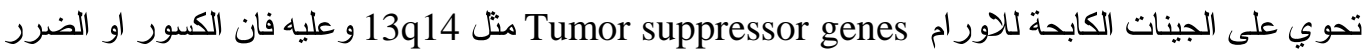

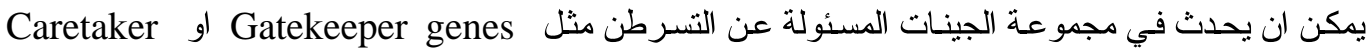
genes

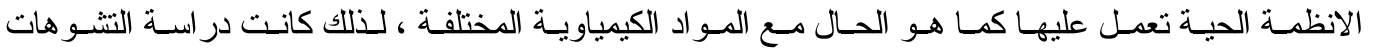

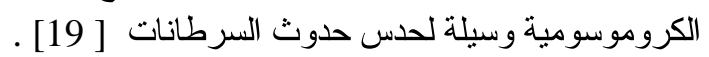

ونتائج تكون النوى الصغيرة في الخلايا اللمفاوية موضحة في الثنكل (2). 


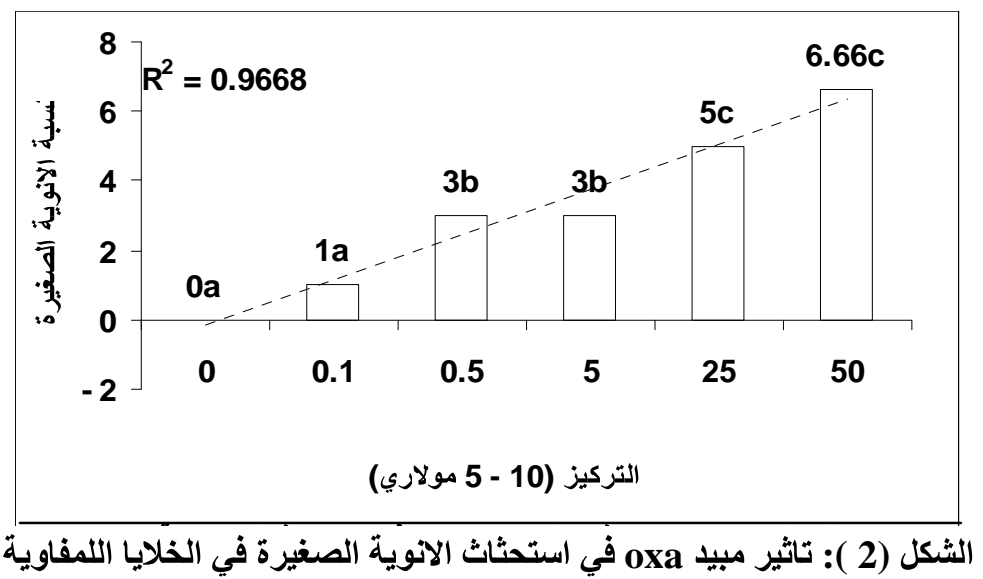

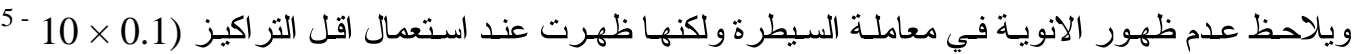

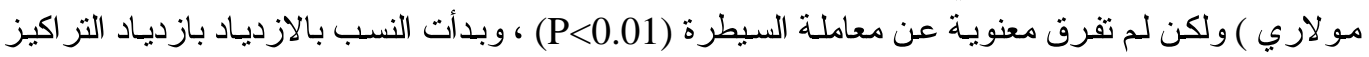

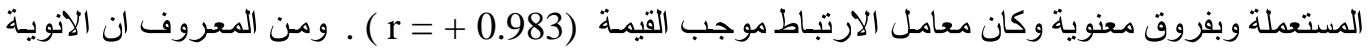

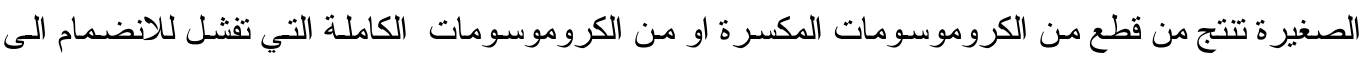

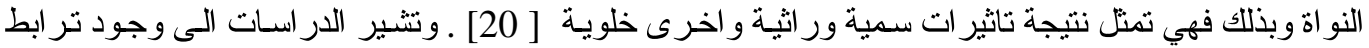

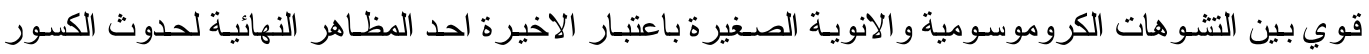

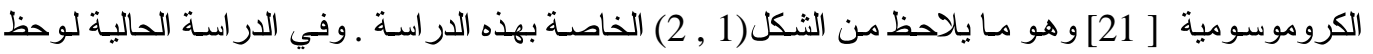

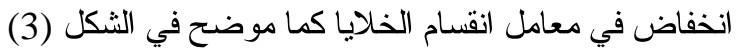

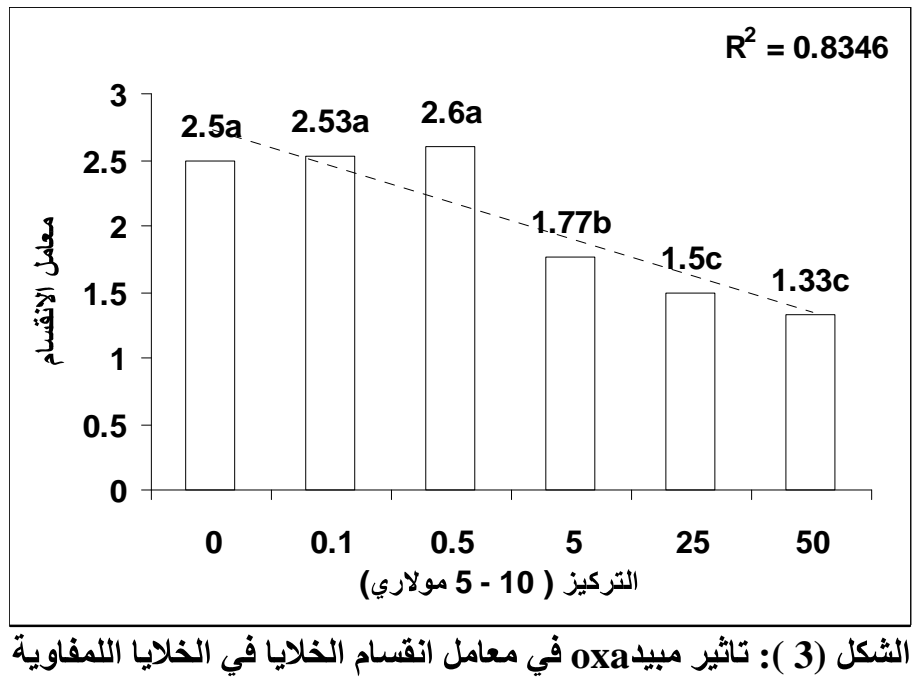

و الملاحظ ان التر اكيز الو اطئة (0.1 و 0.5 × 10 - 5 مو لاري ) لم تؤثر في معامل الانقسام وبقيت الفروق غير

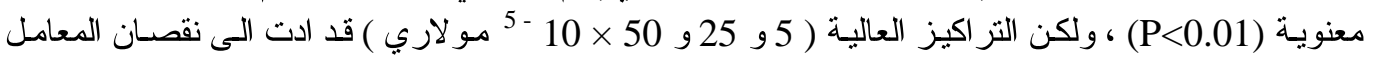

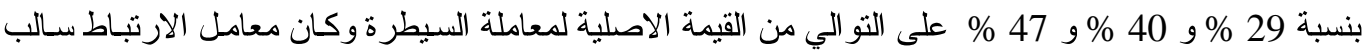

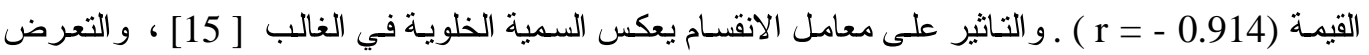

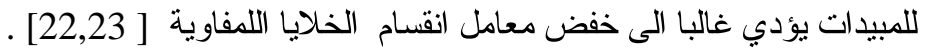

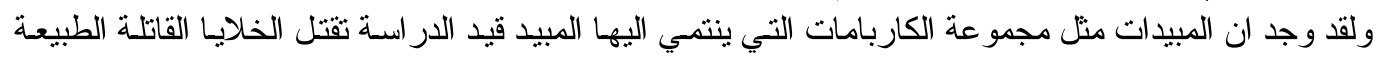

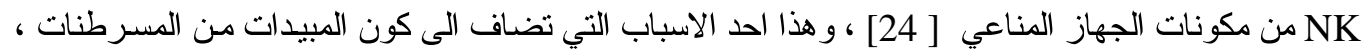

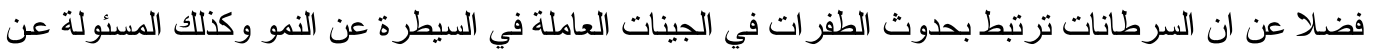

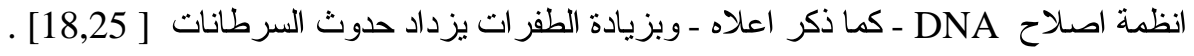


1. WHO (2001). The WHO Recommended Classification of Pesticides by Hazard and Guidelines to Classification 2000 - 2002. Geneva: World Health Organization. Programme on Chemical safety.

2. Paz-y-Mino, C.; G. Bustamante; M. Sanchz and P. Leone (2002). Cytogenetic monitoring in a population occupationally exposed to pesticides in Ecuador. Environ. Health Perspect. 110: 1077 - 1080.

3. Carpenter, D.; K. Acaro and D. Spink (2003). Understanding the human health effects of chemical mixtures. Environ. Health Perspect. 110: 25 - 42.

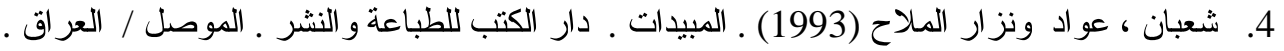

5. Cox, C. (1999). Do pesticides contaminate our reviers, streams and wells? J. Pesti . Reform. 19: $6-8$.

6. Andrade, M.; M. Reyzbal; E. Couelo and F. Vega (2005). Organochlorine pesticides in soils of the horticultural. Can. J. Soil Sci . 85: 273 - 282.

7. Baily , P ; D . Waite; L. Abbott and B. Repley (2005). Residues of DDT and other selected organochlorine agricultural. Can. J. Soil Sci . 85: $256-271$.

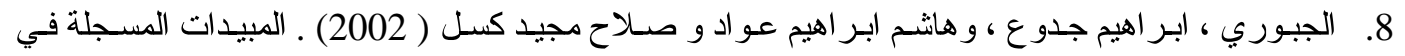

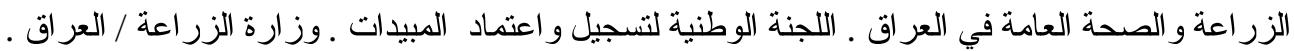

9. Fenech, M . (2000). the in vitro micronucleus technique. Mut . Res. 455: 83 - 93.

10. Benn, P. and A. Perle (1992). Chromosome Staining and Banding Technique. In "Human Cytogenetics" D. Rooney and B. Czpulkowski (Eds . ) . Oxford University Press: UK.

11. Bauchinger, M.; E. Schmid, and J. Dresp (1983). Quantitative analysis of chromosome damage at first division of human lymphocytes after radiation. Rad. Environ Biophys. 22: 225-229.

12. Gohosh, B.; G. Taluker and A. Shorma (1991). Effect of culture media on spontaneous incidence of mitotic index, chromosomal aberration, SCE, and cell cycle in peripheral blood lymphocytes of male and female donors. Cytogenetic . 67: 71-75.

13. Tawn , E . and D. Holdsworth (1992). Mutagen Induced Chromosome Damage in Human Lymphocytes. In "Human Cytogenetics" . D. Rooney and B. Czpulkowski (Eds . ) . Oxford University Press: UK .

14. Duncan, D. (1955) . Multiple range and multiple F- test. Biometric 11:1 - 42

15. Bhalli , J . ; Q . Khan ; A . Haq ; A . Khalid and A. Nasim (2006) . Cytogenetic analysis of Pakistani individuals occupationally exposed to pesticides in a pesticide production industry . Mutagenesis $21: 143-148$.

16. Strachan, T . and A . Read ( 1999) . Human Molecular Genetics . BIOS Scientific Publishers, Ltd .

17. Garry, V .; R. Tarone ; L . Long ; J . Kelly and B . Burroughs (1996 ). Pesticide appliers with mixed pesticide exposure : $\mathrm{G}$ - banded analysis and possible relationship to non- Hodgkin's lymphoma . Cancer Epidemiol . Biomarkers \& Prevent . $5: 11-16$.

18. Goldman , R . and Shields (2003) . Food Mutagens . J . Nutr. 133 : 965 - 973 .

19. Hagmar , L . ; S . Bonassi ; U . Stromberg ; A . Brogger ; L . Knudsen ; H . Norrpa and C . Reuterwell ( 1998). Chromosomal aberrations in lymphocytes predict 
human cancer : a report from the European study group on cytogenetic biomarkers and health . Cancer Res . $58: 4117$ - 4121 .

20. Fenech, M. (1993). The Cytokinesis - blocked micronucleus technique : a detailed description of the method and it's application to genetoxicity studies in human population . Mut . Res . $285: 35-44$.

21. Meng , Z . and B . Zhang (1997). Chromosomal aberrations and micronuclei in lymphocytes of workers at a phosphate fertilizer factory. Mut. Res. 393: 283-288 .

22. Rupa , D .; P . Reddy; K . Sreemannaravana and O . Reddy (1991) . Frequency of sister chromatid exchange in peripheral lymphocytes of male pesticides applicators . Environ . Mol . Mutagen . $18: 136-138$.

23. Pasquini , R .; G . Seassellati-Sforzolini ; G . Angeli ; C . Fatigoni ; S . Manorca ; L . Beneventi ; A . DiGiulio and F . Bauleo (1996) . Cytogenetic biomonitoring of pesticide - exposed farmers in central Italy . J . Environ . Pathol . Toxicol . Oncol . $15: 29-39$.

24. Whalen, M.; B. Longanathan; N. Yamashita and T . Saito ( 2003) . Immunomodulation of human natural killer cell cytotoxic function by triazine and carbamate pesticides, Chem . Biol. Interact. 145: $311-319$.

25. Berg, J. ; J. Tymoczko and L. Stryer (2002). Biochemistry. $5^{\text {th }}$ edition. W .H. Freeman and Company. Oxford, UK. 